\title{
Wave Propagation over a Submerged Bar
}

\author{
L.H. Wiryanto
}

Faculty of Mathematics and Natural Sciences, Institut Teknologi Bandung

Jalan Ganesha 10 Bandung

leo@math.itb.ac.id

\begin{abstract}
Based on small amplitude water theory we determine an exact solution for the amplitude of the reflected and transmitted waves produced when a monochromatic wave hits a submerged bar, a result of importance in coastal engineering. We compare the results obtained with results previously obtained in the long wave length limit and other numerical results. Using the results obtained we determine the optimal width or the bar of prescribed depth.
\end{abstract}

Keywords: linear shallow water equations; monochromatic waves; potential function; reflected waves; submerged bar; transmitted waves.

\section{$1 \quad$ Introduction}

In this paper, we examine the propagation of monochromatic waves over a submerged bar. In particular we determine the reflection and transmission coefficient as a function of the depth and width of the bar. The effect of the bar is to reduce the amplitude of the incident waves, so that such bars, either artificial or natural, are of importance in coastal engineering.

The change of the water depth, from deep to shallower, effects to the waves by increasing the wave amplitude, and the other way around. A bar, submerged beneath water surface, is typical change of depth in a channel. It is often seen as practical application in coastal and ocean engineering, such as beach protector from waves, since it can reduce the wave amplitude. The incoming waves are partly reflected when they pass over the bar. The percentages of the reflected and transmitted waves depend on the dimension of the bar. In order to answer that objective, we solve the governing equation of potential function by variable separation method.

Recently, Pudjaprasetya and Chendra [1] reported their numerical work on wave propagation over parallel bars based on linear shallow water equations. For a single bar, the model can be solved analytically, see for example in Mei [2], and it was confirmed numerically in [1]. Since the model was derived by series expansion, equivalent with approximating the hyperbolic function in the dispersion relation, this causes less accurate in evaluating the reflected and transmitted waves. Groesen and Andonowati [3] show similarity between optic

Received August $20^{\text {th }}, 2009,1^{\text {st }}$ Revised January $6^{\text {th }}, 2010,2^{\text {nd }}$ Revised February $9^{\text {th }}, 2010,3^{\text {rd }}$ Revised February $22^{\text {nd }}, 2010$, Accepted for publication February $24^{\text {th }}, 2010$. 
and water waves based on the same model. Yu and Mei [4] studied waves over a bar by including the effect of the reflection from the beach. Other references can be found by reading the review paper in [5].

In improving the result from shallow water equation, we propose in this paper an alternative approach. The governing equation of the wave over flat bottom is first solved analytically by method of variable separation. The potential function is determined by assuming as multiplication of two functions, each having different variable separately. This method is restricted only for linear boundary conditions, and monochromatic waves. Wiryanto and Anwarus [6] applied the similar method for surface waves over permeable bed. For waves passing a bar, the potential function for different water depth, obtained above, is matched at the position where the water changes the depth discontinuously. As the result, this method is able to determine the formulae for the amplitude of the reflected and transmitted waves. This result can be confirmed to the previous result from shallow water equations by linearizing the formulae.

\section{Problem Definition}

The problem formulated here is water waves propagating, from left to right over a bar, illustrated in Figure 1. We use Cartesian as the coordinates, the $x$-axis is chosen along the undisturbed level of the water surface, and the $y$-axis is perpendicular to the $x$-axis. The bottom channel changes from water depth $h_{2}$ to $h_{1}$, at $x=0$, and similarly at the position $x=L$, the water depth changes from $h_{1}$ to $h_{0}$. It is possible that the water depth before and after the bar is different. A monochromatic wave of frequency $\omega$, and associated wave number $k_{2}$, is normally incident on the step. The wave is partially reflected and transmitted. Assuming potential flow, the surface elevation can be expressed in the form

$$
\eta(x, t)= \begin{cases}a e^{-i\left(k_{2} x-\omega t\right)}+a_{r} e^{-i\left(-k_{2} x-\omega t\right)}, & x<0 \\ b_{t} e^{-i\left(k_{1} x-\omega t\right)}+b_{r} e^{-i\left(-k_{1} x-\omega t\right)}, & 0<x<L \\ c e^{-i\left(k_{0} x-\omega t\right)}, & x>L\end{cases}
$$

where $k_{0}$ and $k_{1}$ are the wave number corresponding to the depth of the water, and the coefficients $a, a_{r}, b_{t}, b_{r}$ and $c$ are the amplitude of the waves. The subscript $t$ denotes transmission, and $r$ denotes reflection. After passing the bar, there is only one type of waves, i.e. transmitted waves. In the regions where wave interaction occurs, we present the transmission and reflection in the same 
wave number, but different sign, indicating the direction of the waves, to the right or to the left. Our aim is to determine the reflection and transmission coefficients $a_{r}, c$ as a function of the bar size. Without loss in generality we choose $a=1$. The reflection and transmission coefficients may be complex to account for phases difference between the waves.

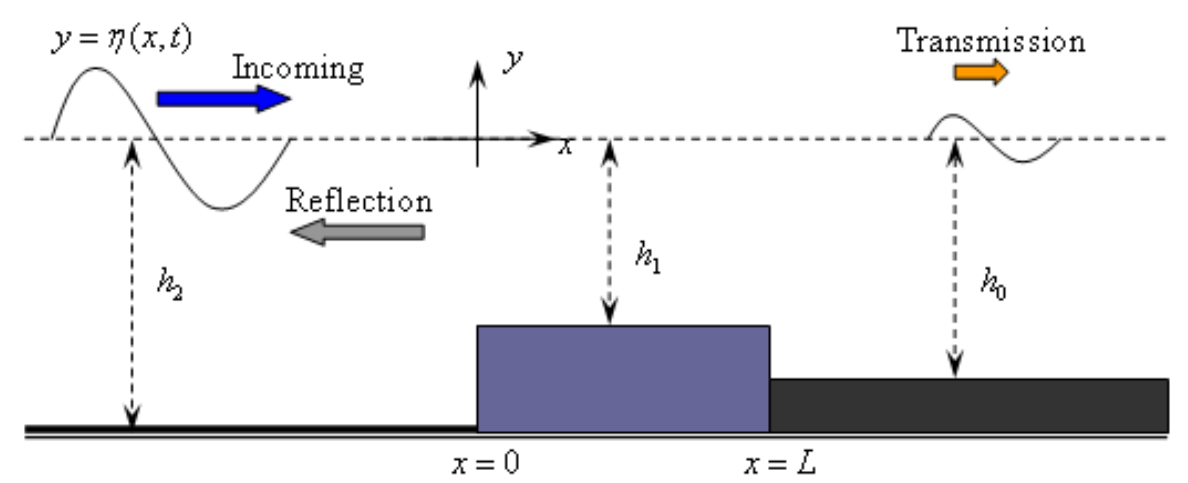

Figure 1 Sketch of the coordinates and the waves over a submerged bar.

Associated with the above description, the governing equation is Laplacian of the potential function

$$
\phi_{x x}+\phi_{y y}=0
$$

followed by boundary conditions along the surface and the bottom. In this case, we only concern with linear conditions,

$$
\begin{aligned}
& \eta_{t}-\phi_{y}=0 \\
& \phi_{t}+g \eta=0
\end{aligned}
$$

on the surface, and

$$
\phi_{y}=0
$$

on the bottom.

\section{Monochromatic Waves over a Flat Bottom}

Before observing monochromatic waves passing over a submerged bar, we present the solution of the potential function for monochromatic waves over a flat bottom. The variable separation is used, and the dispersion relation of the waves is derived in this section. 
In a region having a flat bottom and water depth $h$, we express the potential function as multiplication

$$
\phi(x, y, t)=S(x, t) F(y)
$$

Before determining $\phi$, we first determine $S$ as a specific function. We use condition (4), giving $F(0)=-g \eta / S_{t}$. Since $F(0)$ is constant, $S_{t}$ and $\eta$ must be proportional, that can be written as $\eta=\alpha S_{t}$ for constant $\alpha$. This relation is then integrated with respect to $t$, and we express $\eta$ as monochromatic wave

$$
\eta(x, t)=e^{-i(k x-\omega t)},
$$

giving

$$
\alpha S=\frac{\eta}{i \omega}
$$

The constant of integration is zero, since $\eta$ is proportional to $S$, that can be obtained when condition (3) is used for $\phi$. Without loss of generality we use condition $F(0)=1$, satisfied by $\alpha=-1 / g$, so that we obtain

$$
S(x, t)=\frac{i g}{\omega} \eta(x, t)
$$

Consequently, we obtain $F^{\prime}(0)=\omega^{2} / g$.

The variable separation (6) is then substituted into (2), giving

$$
F^{\prime \prime}-k^{2} F=0 \text {. }
$$

By solving this ordinary differential equation, we obtain the potential function in form of

$$
\phi(x, y, t)=\frac{i \eta}{\omega}\left[\cosh (k y)+\frac{\omega^{2}}{g k} \sinh (k y)\right]
$$

Finally, from the three conditions (3), (4) and (5), there is one condition left that has not been used, i.e. condition (5). When (8) is substituted into (5), we obtain the relation between $\omega$ and the wave number $k$, i.e.

$$
\frac{\omega^{2}}{g k}=\tanh (k h)
$$


In case the wave-length is relatively long compared to the water depth, the tangent hyperbolic function can be approximated linearly as $k h$, and therefore (9) gives

$$
k= \pm \frac{\omega}{\sqrt{g h}}
$$

This shows that there are two type of monochromatic waves traveling in different direction, but the same wave number. This is the reason why we express the incoming and reflected waves as given in (1) in the region $x<0$, similar reason for the region $0<x<L$.

\section{$4 \quad$ Wave Amplitudes}

The problem in wave propagation over a submerged bar is to determine the amplitudes of transmitted and reflected waves for each region having flat bottom, as written in (1). First, the wave number for each region is determined from (9). Since it is nonlinear, we can solve numerically. Then, for knowing the incoming amplitude $a=1$, we determine $a_{r}, b_{t}, b_{r}$ and $c$. This can be done by considering that the waves must continue, especially at $x=0$ and $x=L$. Mathematically, we have relations

$$
\begin{aligned}
& \lim _{x \rightarrow 0^{-}} \eta(x, t)=\lim _{x \rightarrow 0^{+}} \eta(x, t) \\
& \lim _{x \rightarrow L^{-}} \eta(x, t)=\lim _{x \rightarrow L^{+}} \eta(x, t)
\end{aligned}
$$

We use notation superscript $(.)^{-}$as the limit approached from the left side, and

$(.)^{+}$from the other direction. Therefore, (11) and (12) give

$$
\left.\begin{array}{c}
1+a_{r}=b_{t}+b_{r} \\
b_{t} e^{-i k_{1} L}+b_{r} e^{i k_{1} L}=c e^{-i k_{0} L}
\end{array}\right\}
$$

The next condition, that is used in determining the amplitudes, is the continuing of the flux before and after the bar, i.e. at $x=0$ and $x=L$. In evaluating the flux $Q$, we use depth-average velocity, i.e. for water with depth $h$

$$
Q=\int_{-h}^{0} \phi_{x} d y
$$

Therefore, for $x<0$ the flux is 


$$
Q_{2}=\frac{i g \eta_{x}}{k_{2} \omega} q_{2}
$$

where

$$
q_{2}=\sinh \left(k_{2} h_{2}\right)+\frac{\omega^{2}}{g k_{2}}\left(1-\cosh \left(k_{2} h_{2}\right)\right)
$$

The hyperbolic functions can be canceled by using (9), so that we have

$$
q_{2}=\frac{\omega^{2}}{g k_{2}}
$$

Similarly in $0<x<L$, the flux is

and

$$
Q_{1}=\frac{i \eta_{x} \omega}{k_{1}^{2}}
$$

$$
Q_{0}=\frac{i \eta_{x} \omega}{k_{0}^{2}}
$$

in $x>L$. Now, the water mass crossing at $x=0$ must be the same value when we evaluate using $Q_{2}$ and $Q_{1}$, i.e.

$$
\lim _{x \rightarrow 0^{-}} Q_{2}=\lim _{x \rightarrow 0^{+}} Q_{1},
$$

giving

$$
\left(-1+a_{r}\right) k_{1}=\left(-b_{t}+b_{r}\right) k_{2} .
$$

Similar at $x=L$, the flux satisfies

$$
\lim _{x \rightarrow L^{-}} Q_{1}=\lim _{x \rightarrow L^{+}} Q_{0},
$$

giving

$$
\left(b_{t} e^{-i k_{1} L}-b_{r} e^{i k_{1} L}\right) k_{0}=c e^{-i k_{0} L} k_{1}
$$

When Equations (13), (14) and (15) are solved; we obtain

$$
b_{r}=\frac{2\left(k_{1}^{2}-k_{0} k_{1}\right) e^{-i k_{1} L}}{\left(k_{1}-k_{2}\right)\left(k_{1}-k_{0}\right) e^{-i k_{1} L}-\left(k_{1}+k_{2}\right)\left(k_{0}+k_{1}\right) e^{i k_{1} L}}
$$




$$
\begin{aligned}
& b_{t}=\frac{2 k_{1}-\left(k_{1}-k_{2}\right) b_{r}}{k_{1}+k_{2}} \\
& c=e^{i k_{0} L}\left(b_{t} e^{-i k_{1} L}+b_{r} e^{i k_{1} L}\right)
\end{aligned}
$$

All amplitudes are in complex number. These are then returned into (1), and the analytic solution of the surface waves $\eta$ can be taken as the real or imaginary part of (1). The wave numbers, $k_{0}, k_{1}$ and $k_{2}$, are first calculated by solving (9) corresponding to the depth of the water, and we then use them to determine $b_{r}, b_{t}$ and $c$ by (16)-(18). The amplitude reduction of the transmitted wave can be compared between the modulus of $c$ and the amplitude of the incoming wave, in this case we define $a=1$. The length $L$ plays an important role in determining the amplitude reduction, and appears in modulus $|c|$ as part of trigonometric functions. In observing the effect of $L$ to the transmitted amplitude, we use numerical calculation to reduce the complexity in determining the wave numbers and the amplitudes (16)-(18).

We perform the surface profile in Figure 2, corresponding to waves propagating over a submerged bar for $h_{1}=1.0, h_{0}=h_{2}=3.5, L=6.0$, and $\omega=2.0$, $g=10$. The surface wave is defined as the real part of (1), and we plot for some different $t$ in the same plane by shifting the curves upward for larger $t$. The interaction between the incoming and reflected waves in $x<0$ produces surface with changing the amplitude. This can be compared with the profile in $x>L$, performing traveling waves. The location of the bar is indicated by grey region. The amplitudes of the reflected and transmitted waves are $\left|a_{r}\right|=0.337$ and $|c|=0.941$

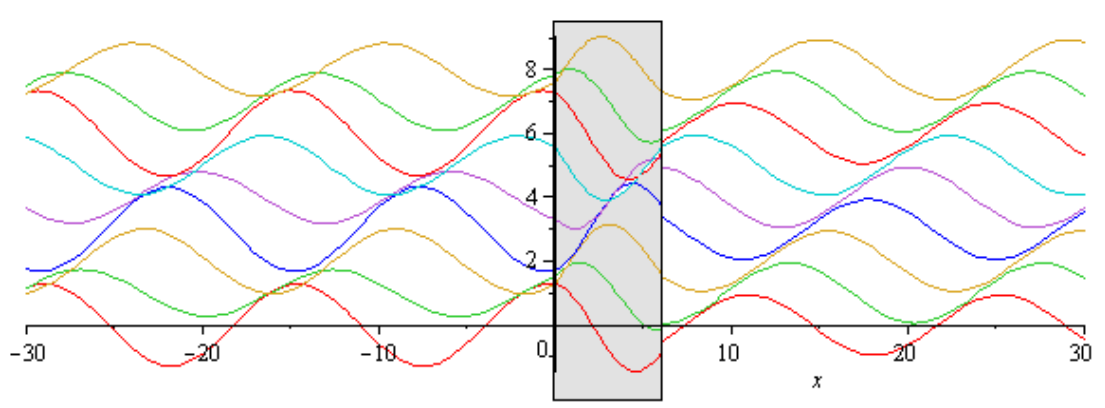

Figure 2 Plot of waves propagating over a submerged bar. 
If the wave length is relatively large compared with the water depth then the dispersion relationship can be linearlized to give the wave number (10). When this approximation is applied to (16)-(19), we have formulation to calculate the wave amplitudes as obtained in derivation using shallow water equations, such as presented in [1]. As the comparison, we calculate the reflected and transmitted using the above parameters giving $\left|a_{r}\right|=0.376$ and $|c|=0.927$.

For some other values $L$, we calculate the amplitude of the transmitted waves after passing the submerged bar, i.e. $|c|$, and we plot between both quantities shown in Figure 3, for our method and shallow water equations (SWE), as indicated on the curve. Note that the data for SWE is evaluated based on the linearized dispersion relation (10). In the range of our calculations $L \in[1,8.5]$, we obtain two minimum values of $|c|$, called optimal value $L_{\min }$, i.e. at $L_{\min }=2.2$ and 6.95. These numbers are slightly different with the calculation using shallow water equations, obtaining $L_{\min }=2.5$ and 7.5. Between two values $L_{\min }$ for each case, the smaller $L_{\min }$ is more efficient than the second one as we obtain smaller amplitude of the transmitted wave. Hence, we calculate $L_{\min }$ for various $h_{1}$. We obtain that increasing $h_{1}$ is followed by increasing $L_{\text {min }}$, as shown in Figure 4.

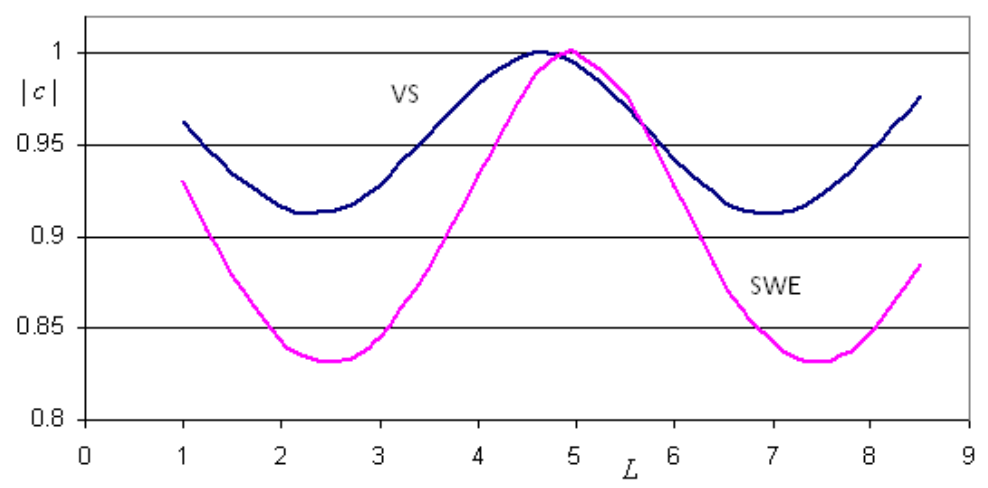

Figure 3 Plot of $L$ versus $|c|$ from shallow water equations (SWE) and variable separation (VS).

For different values between $h_{0}$ and $h_{2}$, the surface profile can be calculated from our model. In case the water depth before the bar is deeper than after the bar, $h_{2}>h_{0}$ but $h_{0}>h_{1}$, the waves are transmitted with larger amplitude than 
before, i.e. $h_{2}=h_{0}$. It is possible $|c|$ greater than the amplitude of the incoming waves for relatively long of the bar width $L$. In Figure 5, we show plot of surface profile presenting wave propagation over a submerged bar with $h_{1}=1.4, h_{0}=2.5, h_{2}=3.5, L=5.0$, and $\omega=2.0, g=10$. The value of $L$ is larger than $L_{\min }=2.3$, and it has the amplitude of the transmitted waves $|c|=1.0434$, almost the same amplitude of the incoming waves and small reflected waves. Hence, the profile is like propagation without submerged bar.

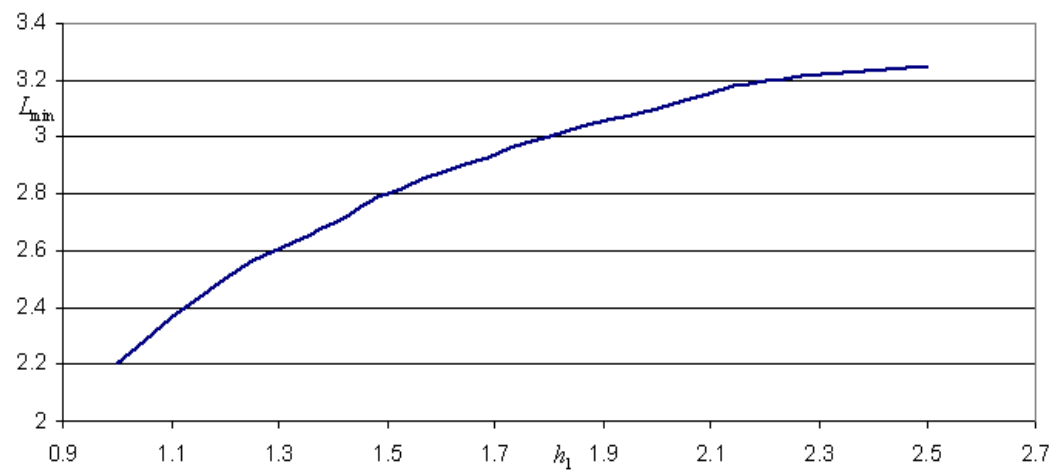

Figure 4 Plot of $h_{1}$ versus $L_{\min }$.

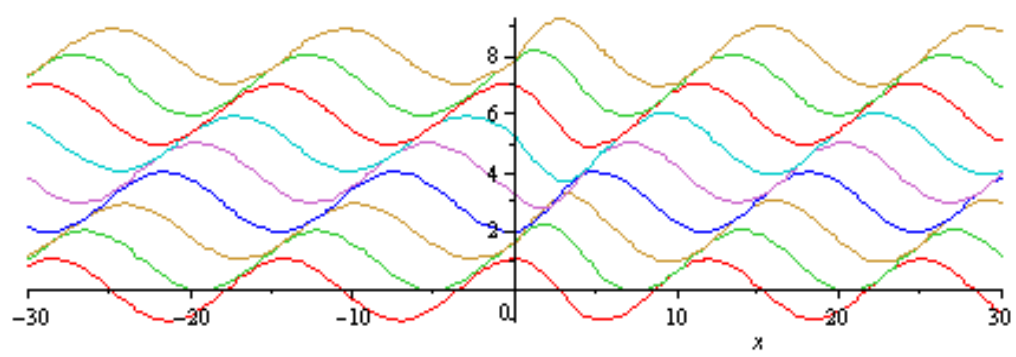

Figure 5 Plot of waves propagating over a submerged bar with $h_{1}=1.4$, $h_{0}=2.5, h_{2}=3.5, L=5.0$.

\section{$5 \quad$ Conclusion}

An analytic solution of wave propagation passing over a bar has been solved by variable separation. Potential function was determined from Laplace equation and linear boundary conditions of water on flat bottom for monochromatic waves. This formulation was then applied to different water depth to obtain the amplitudes of transmitted and reflected waves. As the results, we found that our 
solution confirms to the results derived from linear shallow water equations when we linearize the hyperbolic functions involved in our formulation. The optimal dimension of the bar, that gives the minimum amplitude of the transmitted waves, is obtained numerically as the wave number has to be calculated from nonlinear dispersion relation and to reduce the complexity of the formula. Comparison was made to the previous result for long wave-length limit.

\section{Acknowledge}

The author is grateful to Bandung Institute of Technology and Ministry of Department of Education for supporting of the research of this paper, under contract No. 164/SP2H/PP/DP2M/V/2009. The author also thank to Dr. S.R. Pudjaprasetya for useful discussion during solving the problem.

\section{References}

[1] Pudjaprasetya, S.R., Chendra, H.D., An Optimal Dimension of Submerged Parallel Bars as a Wave Reflector, Bull. Malay. Math. Sci. Soc., 32(1), 55-62, 2009.

[2] Mei, C.C., The Applied Dynamics of Ocean Surface Waves, World Scientific, 1989.

[3] Groesen, E., Andonowati, Similarities between Optical and Surface Water Waves, J. Indones. Math. Soc., 8(3), 1-8, 2002.

[4] Yu, J., Mei, C.C., Do Longshore Bars Shelter The Shore?, J. Fluid Mech., 404, 251-268, 2000.

[5] Mei, C.C., Liu, P.L.F., Surface Waves and Coastal Dynamics, Annual Review of Fluid Mechanics, 25, 215-240, 1993.

[6] Wiryanto, L.H., Anwarus, Monochromatic Waves over Permeable Bed, Proc. 5th Asian Math. Conf., Kuala Lumpur, 617-622, 2009. 